\title{
Using Patchy Particles to Shed New Light on the Autocatalytic Aggregation of Soft Matter ${ }^{+}$
}

\author{
Silvia Corezzi ${ }^{1, *}$, Francesco Sciortino ${ }^{2}$ and Cristiano De Michele ${ }^{2}$ \\ Department of Physics and Geology, University of Perugia, I-06123 Perugia, Italy \\ 2 Department of Physics, 'Sapienza' University of Rome, I-00185 Rome, Italy \\ * Correspondence: silvia.corezzi@unipg.it \\ + Presented at the 37th International Symposium on Dynamical Properties of Solids (DyProSo 2019), Ferrara, \\ Italy, 8-12 September 2019.
}

Published: 5 September 2019

Autocatalysis, i.e., the speeding up of a reaction through the very same molecule which is produced, is common in chemistry, biophysics, and material science. Despite the pervasiveness of autocatalytic phenomena in nature and technology, autocatalytic aggregation has so far remained out of reach of realistic numerical simulations. Rate-equation-based approaches are often used to model the time dependence of products, but the key physical mechanisms behind the reaction cannot be properly recognized and then taken into account. On the contrary, patchy particles are designed learning from nature and can be thought of as an archetype of real monomers, but so far they completely lack autocatalysis. Here, building on previous studies on the subject [1-4], we report on a patchy particle model inspired by a bicomponent reactive mixture and endowed with adjustable autocatalytic ability [5]. Such a coarse-grained model captures all general features of an autocatalytic aggregation process that takes place under controlled and realistic conditions, including crowded environments. Simulation reveals that a full understanding of the kinetics involves an unexpected effect that eludes the chemistry of the reaction, and which is crucially related to the presence of an activation barrier. The resulting analytical description can be exported to real systems, as confirmed by experimental data on epoxy-amine polymerizations, solving a long-standing issue in their mechanistic description.

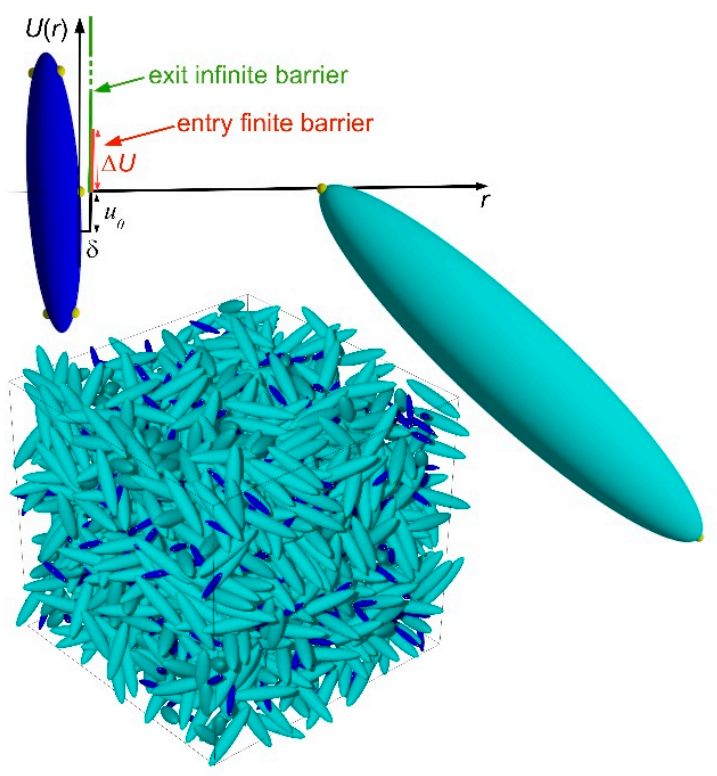




\section{References}

1. Corezzi, S.; De Michele, C.; Zaccarelli, E.; Fioretto, D.; Sciortino, F. A molecular dynamics study of chemical gelation in a patchy particle model. Soft Matter 2008, 4, 1173-1177.

2. Corezzi, S.; De Michele, C.; Zaccarelli, E.; Tartaglia, P.; Sciortino, F. Connecting irreversible to reversible aggregation: Time and temperature. J. Phys. Chem. B 2009, 113, 1233-1236.

3. Corezzi, S.; Fioretto, D.; De Michele, C.; Zaccarelli, E.; Sciortino, F. Modeling the crossover between chemically and diffusion-controlled irreversible aggregation in a small-functionality gel-forming system. J. Phys. Chem. B 2010, 114, 3769-3775.

4. Corezzi, S.; Fioretto, D.; Sciortino, F. Chemical and physical aggregation of small-functionality particles. Soft Matter 2012, 8, 11207-11216.

5. Corezzi, S.; Sciortino, F.; De Michele, C. Exploiting limited valence patchy particles to understand autocatalytic kinetics. Nat. Commun. 2018, 9, 2647.

(C) 2019 by the authors. Licensee MDPI, Basel, Switzerland. This article is an open access article distributed under the terms and conditions of the Creative Commons Attribution (CC BY) license (http://creativecommons.org/licenses/by/4.0/). 